\title{
Oral Cavity and Pharynx Cancer Incidence Trends by Subsite in the United States: Changing Gender Patterns
}

\author{
Linda Morris Brown, ${ }^{1}$ David P. Check, ${ }^{2}$ and Susan S. Devesa ${ }^{2}$ \\ ${ }^{1}$ Statistics and Epidemiology Group, RTI International, 6110 Executive Boulevard, Suite 902, Rockville, MD 20852-3907, USA \\ ${ }^{2}$ Division of Cancer Epidemiology and Genetics, National Cancer Institute, National Institutes of Health, \\ Department of Health and Human Services, Bethesda, MD 20892, USA
}

Correspondence should be addressed to Linda Morris Brown, lindabrown@rti.org

Received 15 December 2011; Revised 18 January 2012; Accepted 18 January 2012

Academic Editor: Dong Moon Shin

Copyright (C) 2012 Linda Morris Brown et al. This is an open access article distributed under the Creative Commons Attribution License, which permits unrestricted use, distribution, and reproduction in any medium, provided the original work is properly cited.

\begin{abstract}
Objective. To evaluate oral cavity and pharynx cancer (OCPC) patterns by gender. Methods. We used Surveillance, Epidemiology, and End Results program data for 71,446 cases diagnosed during 1975-2008 to classify OCPC by anatomic subsite as potentially HPV-related or not, with oral tongue cancer considered a separate category. Results. Total OCPC rates among men were 2-4 times those among women. Among whites, total OCPC rates rose in the younger age groups due to substantial increases in successive birth cohorts for HPV-related cancers, more rapid among men than women, and oral tongue cancers, more rapid among women than men. Among blacks, total OCPC rates declined among cohorts born since 1930 reflecting the strong downward trends for HPV-unrelated sites. Among Hispanics and Asians, HPV-unrelated cancer rates generally declined, and oral tongue cancer rates appeared to be converging among young men and women. Conclusions. Decreases in total OCPC incidence reflect reductions in smoking and alcohol drinking. Rising HPV-related cancers among white men may reflect changing sexual practices. Reasons for the increasing young oral tongue cancer rates are unknown, but the narrowing of the gender differences provides a clue.
\end{abstract}

\section{Introduction}

In the United States, incidence rates of oral cavity and pharynx cancer (OCPC) have been decreasing at about $1 \%$ per year over the past decade, with some differences according to race, sex, and subsite $[1,2]$. Tobacco use is the major risk factor for these cancers; heavy alcohol drinking is an independent risk factor and appears to enhance the effect of smoking [3]. The attributable risk for tobacco and alcohol use has been estimated at $80 \%$ to $90 \%$ among US white and black men [3]. Dietary factors, particularly consumption of fruits and vegetables, have been consistently associated with reduced risks of OCPC [4]. Oral human papillomavirus (HPV), particularly HPV 16, is a newly recognized cause of a subset of OCPC, responsible for $40-80 \%$ of oropharyngeal squamous cell cancers [5-7]. Rising incidence rates for certain subsites within the oral cavity and pharynx, notably the base of tongue, tonsil, and oropharynx related to HPV infection, have been reported in the US particularly among young white men using data from the Surveillance,
Epidemiology, and End Results (SEER) program [7-14] and National Program of Cancer Registries [15]. Rising incidence rates of oral tongue cancer, a subsite not related to HPV infection, also have been reported, especially among young white women [16]. To better understand the US incidence patterns, especially differences by gender, our analysis updates and extends earlier descriptive studies $[11,12]$ that classified OCPC by anatomic subsite into presumed HPVrelated or HPV-unrelated oral cavity and pharynx cancers by incorporating cases diagnosed through 2008, considering oral tongue cancer not related to HPV as a separate category, including data for Hispanic whites, Asian/Pacific Islanders (Asian/PIs), and American Indian/Alaskan Natives (AI/ANs) as well as blacks and non-Hispanic whites, and presenting age-specific male/female ratios by year of birth.

\section{Methods}

Data from population-based registries in the National Cancer Institute's Surveillance, Epidemiology, and End 
Results (SEER) program [17, 18] were used to calculate incidence rates of invasive squamous cell carcinoma (SCC) (International Classification of Diseases for Oncology, 3rd edition (ICD-O-3) morphology codes 8050-8084) of the oral cavity (ICD-O-3 topography codes C01.9-C06.9) and pharynx (codes C09.0-C10.9, C12.9-C14.8) (excluding lip, salivary glands, and nasopharynx) [19]. Like the classification based on anatomic subsite and etiologic relationship with HPV used in two recent publications [11, 12], we categorized OCPC subsites as presumed HPV-related or not and then considered HPV-unrelated oral tongue separately. Specifically, the HPV-related sites were C01.9base of tongue, NOS (not otherwise specified); C02.4lingual tonsil; C09.0-tonsillar fossa; C09.1-tonsillar pillar; C09.8-overlapping lesion of tonsil; C09.9-tonsil, NOS; C10.0-vallecula; C10.1-anterior surface of epiglottis; C10.2lateral wall of oropharynx; C10.3-posterior wall of oropharynx; C10.4-branchial cleft; C10.8-overlapping lesion of oropharynx; C10.9-oropharynx, NOS; C14.2-waldeyer ring. The HPV-unrelated (except oral tongue) sites were: C03.0upper gum; C03.1-lower gum; C03.9-gum, NOS; C04.0anterior floor of mouth; C04.1-lateral floor of mouth; C04.8overlapping lesion of floor of mouth; C04.9-floor of mouth, NOS; C05.0-hard palate; C05.1-soft palate, NOS; C05.2-uvula; C05.8-overlapping lesion of palate; C05.9-palate, NOS; C06.0-cheek mucosa; C06.1-vestibule of mouth; C06.2-retromolar area; C06.8-overlapping lesion of other and unspecified mouth; C06.9-mouth, NOS; C12.9-pyriform sinus; C13.0-postcricoid region; C13.1-aryepiglottic fold, hypopharyngeal; C13.2-posterior wall of hypopharynx; C13.8-overlapping lesion of hypopharynx; C13.9-hypopharynx, NOS; C14.0-pharynx, NOS; C14.8-overlapping lesion of lip, oral cavity and pharynx. Lastly, the HPV-unrelated oral tongue sites were C02.0-dorsal surface of tongue, NOS; C02.1-border of tongue; C02.2-ventral surface of tongue, NOS; C02.3-anterior $2 / 3$ of tongue, NOS; C02.8-overlapping lesion of tongue; $\mathrm{C} 02.9$-tongue, NOS.

Data from the original nine SEER registries (SEER 9) for cases diagnosed during the 34-year period from January 1, 1975, through December 31, 2008, were available for whites and blacks. These nine registries are located in the metropolitan areas of Atlanta, Detroit, San-Francisco-Oakland, and Seattle-Puget Sound and the states of Connecticut, Hawaii, Iowa, New Mexico, and Utah. For cases diagnosed during 1992-2008, data were available for the racial groups of whites, blacks, Asian/PIs, and AI/ANs and whether of Hispanic origin in the SEER 13, which includes the SEER 9 plus four additional areas, the California metropolitan areas of San Jose-Monterey and Los Angeles, rural Georgia, and Alaska Natives in Alaska. In the recent years 2004-2008, the SEER 9 and 13 registries accounted for about $10 \%$ and $14 \%$ of the US population, respectively [1]. All SEER registries annually meet the Gold Standard Registry Certification from the North American Association of Central Cancer Registries, Inc. for completeness (at least 95\%), accuracy ( $<3 \%$ of cases identified through death certificates only), and timeliness of data. Age-adjusted incidence rates per 100,000 person-years of OCPC (using the 2000 US standard) were calculated for each subsite category (total, HPV-related,
HPV-unrelated, and oral tongue) by sex and race/ethnicity using SEER*Stat [20]. All 95\% confidence intervals (CIs) were estimated using the Tiwari modification [21]. Incidence rate ratios (IRRs) were calculated using females as the referent group.

Temporal trends in age-adjusted incidence rates using five- or six-year time periods 1975-79, 1980-85, 1986-91, 1992-97, 1998-2003, and 2004-2008 were plotted according to year of diagnosis by race/ethnicity, subsite category, and sex. Temporal trends in age-specific rates were plotted according to year of birth to facilitate visual comparison of rates. The year of birth was derived by subtracting age at diagnosis (midpoint of age group, i.e., 35 for age group 2544,50 for age group 45-54, 60 for age group 55-64, and 77.5 for age group 65+) from calendar year of diagnosis (midpoint of the calendar period); the cohorts are referred to by their approximate midyear of birth. Only rates based on at least 10 cases were presented. Temporal trends were plotted so that a slope of 10 degrees represented a change of $1 \%$ per year (i.e., 40 years on the horizontal axis is the same length as one logarithmic cycle on the vertical axis) [22]. Estimates of annual percent changes (EAPCs) were calculated for the period 1985-2008 using weighted least squares.

\section{Results}

There were 27,767 cases (18,573 men and 9,194 women) of OCPC diagnosed among whites and blacks during 19751991 in the SEER 9 and 43,679 cases (30,068 men and 13,611 women) among whites (non-Hispanic), blacks, Hispanics (white), Asian/PIs, and AI/ANs during 1992-2008 in the SEER 13 (Table 1). Total OCPC rates among men were 24 times those among women across each race/ethnicity and time period. Male/female (MF) IRRs for 1992-2008 SEER 13 were highest among blacks for each subsite (IRR $=2.60$ 4.32) and lowest among Asian/PIs (IRR = 2.39) for total, whites for HPV-related (IRR $=3.98)$ and HPV-unrelated $(\mathrm{IRR}=2.16)$, and $\mathrm{AI} / \mathrm{ANs}$ for oral tongue $(\mathrm{IRR}=0.88$, the only IRR not significantly higher than 1.0). The MF IRR for HPV-related cases (recent range 3.98-4.47) was higher than that for HPV-unrelated cases (recent range 2.16-3.37) in all instances except 1975-1991 SEER 9 blacks. The MF IRR for oral tongue (recent range 0.88-2.77) was smaller than that for other HPV-unrelated cases among each racial/ethnic group. Among whites and blacks in the SEER 9, the MF IRRs increased from 1975-1991 to 1992-2008 for HPV-related sites, but declined for HPV-unrelated sites and oral tongue.

Trends in the age-adjusted OCPC incidence rates by race/ethnicity (whites (non-Hispanic), blacks, Hispanics (white), and Asian/PIs), subsite category, and gender in the SEER 13 during 1992-2008 are presented in Figure 1. For historical purposes, trend data for whites and blacks in the SEER 9 are also shown for 1975-2008. As seen in Figure 1, the two sets of rates in recent years were similar except for oral tongue among blacks where the rates were somewhat higher in the SEER 13 than the 9. Because the rates are based on larger numbers of cases and thus are more stable in the SEER 13 than the SEER 9, we will focus on them. 


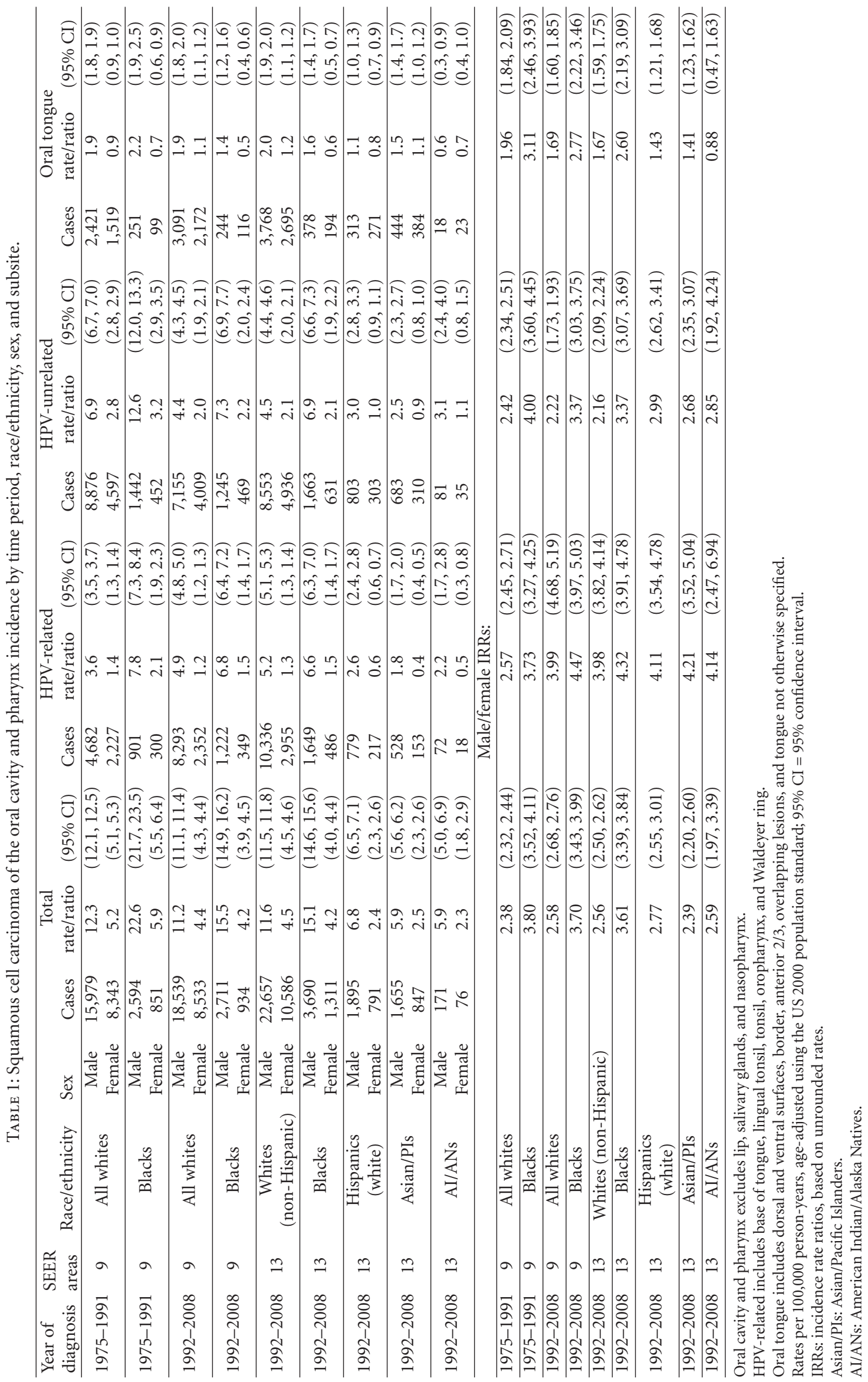



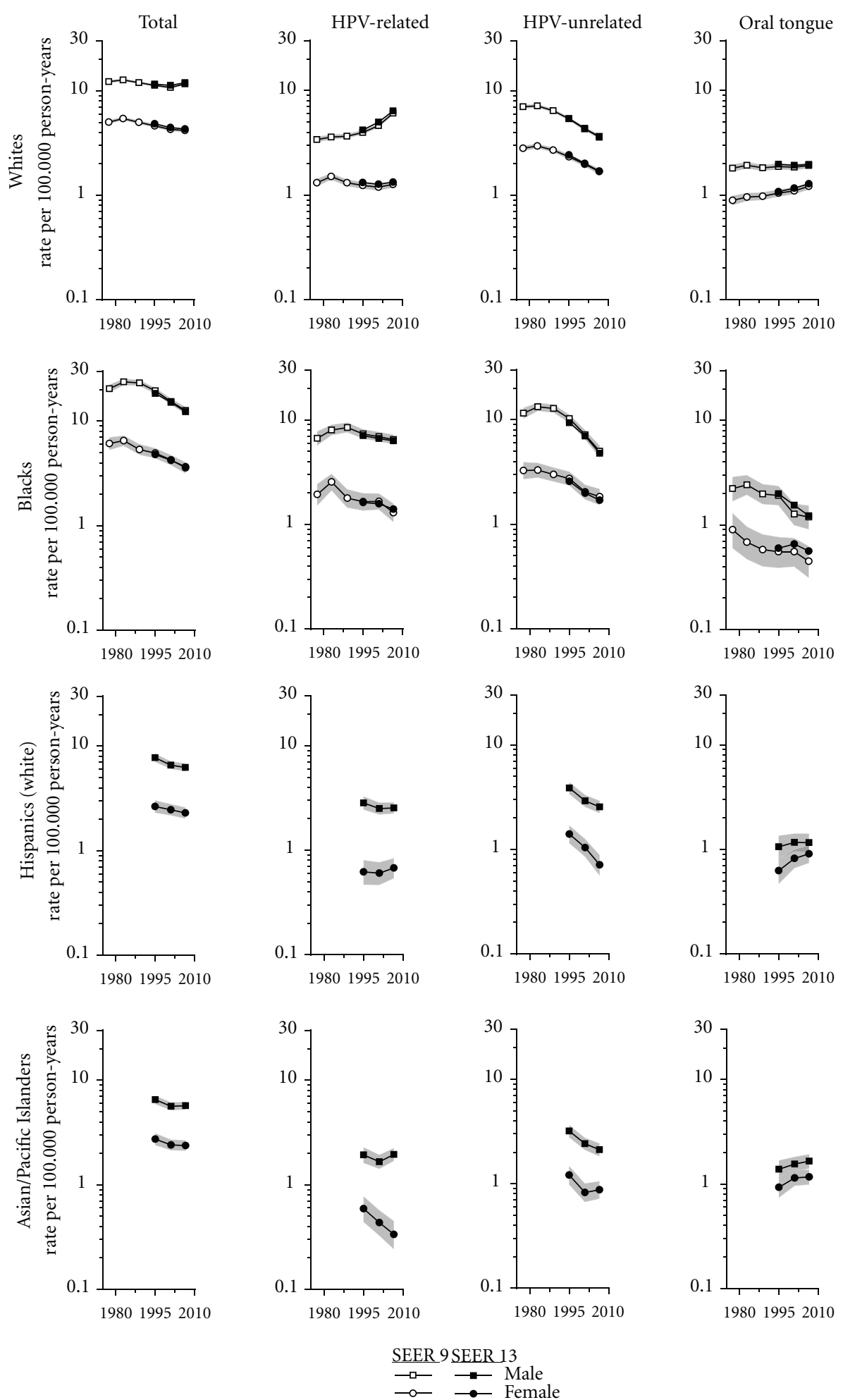

FIgURE 1: Trends in age-adjusted (2000 US standard) squamous cell carcinoma of the oral cavity and pharynx incidence rates by race/ethnicity, subsite, and gender for whites and blacks SEER 9 1975-1979 to 2004-2008 and for whites (non-Hispanic), blacks, Hispanics (white), and Asian/PIs SEER 13 1992-1997 to 2004-2008 according to year of diagnosis (excludes lip, salivary glands, and nasopharynx). Shaded bands portray $95 \%$ confidence intervals. 
Furthermore, it is increasingly important to distinguish nonHispanics and Hispanics among whites because their rates are quite different, and the population proportions have been changing over time. Thus, we will use the term whites to refer to whites in the SEER 9 1975-1991 and white non-Hispanics in the SEER 13 1992-2008, blacks in the SEER 9 early years and SEER 13 recent years, and Hispanics for Hispanic whites and Asians for Asian/PIs in the SEER 13 recently. Among whites, total rates for men peaked at 12.7 per 100,000 personyears in $1980-1985$ before declining gradually $(11 \%)$ to 11.3 in 1998-2003 and then rose 5\% to 12.0 in 2004-2008. Rates among women also peaked in 1980-1985 at 5.4 and declined more rapidly $(20 \%)$ to 4.3 in 2004-2008. Among blacks, total rates for both men and women peaked during 19801985 and then declined dramatically until 2004-2008, by $48 \%$ from 23.7 to 12.3 among males and by $43 \%$ from 6.5 to 3.7 among females. During the recent 17 -year period, total rates decreased significantly among all racial/ethnic/gender groups except white men.

HPV-related rates among white men rose $88 \%$ from 3.4 in $1975-1979$ to 6.4 in 2004-2008, more rapidly in recent years than earlier, while HPV-unrelated rates declined $50 \%$ from 7.2 in 1980-1985 to 3.6 in 2004-2008 (Figure 1). Among the other race/sex groups, rates for HPV-related sites generally decreased or changed little. HPV-unrelated rates declined sharply among all race/sex groups, similar to white males. Rates decreased from 1980-1985 to 2004-2008 among white females by $43 \%$ from 3.0 to 1.7 and among blacks even more rapidly, by $64 \%$ among men from 13.3 to 4.8 , and by $48 \%$ among females from 3.3 to 1.7 . Rates also declined rapidly among Hispanics (men 34\%, women 49\%) and Asians (men 34\%, women 28\%) during 19922008. Oral tongue cancer rates among white men varied little over time, whereas rates in white women increased $44 \%$ from 0.9 in 1975-1979 to 1.3 in 2004-2008. Rates rose more rapidly among females than males among Hispanics (men $10 \%$, women $45 \%$ ) and Asians (men 19\%, women $26 \%$ ). In contrast, rates among blacks declined 50\% for males (from 2.4 in 1980-1985 to 1.2 in 2004-2008) and 33\% for females (from 0.9 in $1975-1979$ to 0.6 in 2004-2008). The EAPCs from 1985 to 2008 among white males were $+2.9 \%$ for HPV-related cancers and $-3.1 \%$ for HPV-unrelated cancers, among white females $-2.7 \%$ for HPV-unrelated cancers and $+1.1 \%$ for oral tongue cancers, and among blacks for HPVunrelated cancers $-4.9 \%$ and $-2.9 \%$ for males and females, respectively.

The MF IRRs among whites doubled from 2.4 to 4.8 for HPV-related cancers declined modestly from 2.4 to 2.1 for HPV-unrelated cancers, and moderately from 2.0 to 1.5 for oral tongue cancers. Among blacks, the decrease in MF IRR for HPV-unrelated cancers was quite rapid, from 4.0 to 2.8.

Among whites, total rates rose in the youngest age group among men and women due to substantial increases in successive birth cohorts for HPV-related cancers, more rapid among men than women, and oral tongue cancers, more rapid among women than men (Figure 2). The HPV-related rates among men aged 25-44 years more than tripled from 0.4 per 100,000 man-years in the 1942 birth cohort to 1.5 in the 1972 cohort, and rates among men aged 45-54 years more than doubled from 5.3 for the 1939 birth cohort to 12.8 for the 1956 birth cohort; rates also rose among men 55-64 from 12.5 for the 1923 cohort to 23.0 for the 1946 cohort. The EAPCs among men aged 25-64 each were $>3 \%$. Among women, HPV-related rates for the older age groups did not vary greatly in successive birth cohorts. For HPV-unrelated cancers, rates among white men declined $67 \%$ (from 0.9 to 0.3 ), $54 \%$ (from 9.2 to 4.2 ), $59 \%$ (from 23.9 to 9.7 ), and $42 \%$ (from 28.6 to 16.5 ) among those aged $25-44,45-54,55-$ 64 , and 65+ years, respectively; the EAPCs ranged between $-3.1 \%$ and $-4.0 \%$ among all but the oldest. Rates among women also declined $37 \%$ (from 0.27 to 0.17 ), $66 \%$ (from 4.1 to 1.4 ), $66 \%$ (from 9.8 to 3.3 ), and $24 \%$ (from 12.2 to 9.3 ), respectively. Oral tongue cancer rates among women aged 25-44 years more than tripled at $+3.4 \%$ per year from 0.2 per 100,000 woman-years in the 1942 birth cohort to 0.7 in the 1972 cohort where rates approached those for young men, among whom rates increased $71 \%$ over the 30 -year time period. Oral tongue rates also rose among women aged 4554 years born since 1940 but not among men; rates for men or women in the older age groups did not change greatly.

Among blacks, total rates declined among cohorts born since at least 1930 reflecting the strong downward trends among men and women especially for HPV-unrelated sites (Figure 2). Black men aged 25-44 experienced the most rapid decrease in rates for HPV-unrelated sites across successive birth cohorts, $82 \%$ (from 4.5 to 0.8 ); and rates among males aged 45-54, 55-64, and 65+ years also declined impressively, $76 \%$ (from 28.3 to 6.8 ), $74 \%$ (from 51.1 to 13.4 ), and $43 \%$ (33.5 to 19.2), respectively. The corresponding EAPCs were $-7.4 \%$ among those aged $25-44$, and $-6.8 \%,-5.5 \%$, and $-2.7 \%$ among the oldest. Rates decreased notably among women aged 25-44 (78\%), 45-54 (76\%), and 55-64 (64\%), but not among women aged 65+. Oral tongue cancer rates among men generally declined across successive birth cohorts except for the older age groups where rates were relatively stable; in recent years among the youngest age group, the male predominance was no longer apparent. Of note, there was no suggestion of rapid increases in rates of HPV-related or oral tongue cancers among young blacks as seen among whites.

Among Hispanics and Asians, total OCPC trends were not consistent across the genders or age groups. However, rates of HPV-unrelated cancer generally declined across successive birth cohorts for both genders. Oral tongue cancer rates appeared to be converging among young men and women of both groups.

The MF IRRs for total among whites remained about 2 among those born prior to 1940, after which they rose to 2.93.8 among those born during the 1950s and then declined to 2.3 among those born around 1970 (Figure 3). MF IRRs for HPV-related cases were higher, hovering between 2 and 3 among those born prior to 1930 , after which they rose to almost 6 among those aged 45-64 years born during the late 1940s-1950s; among those aged 25-44, the IRR rose from 1.9 among those born in the early 1940 s to about 4 among those born since the mid-1950s. The MF IRRs for HPVunrelated cases varied less by age group and year of birth, 

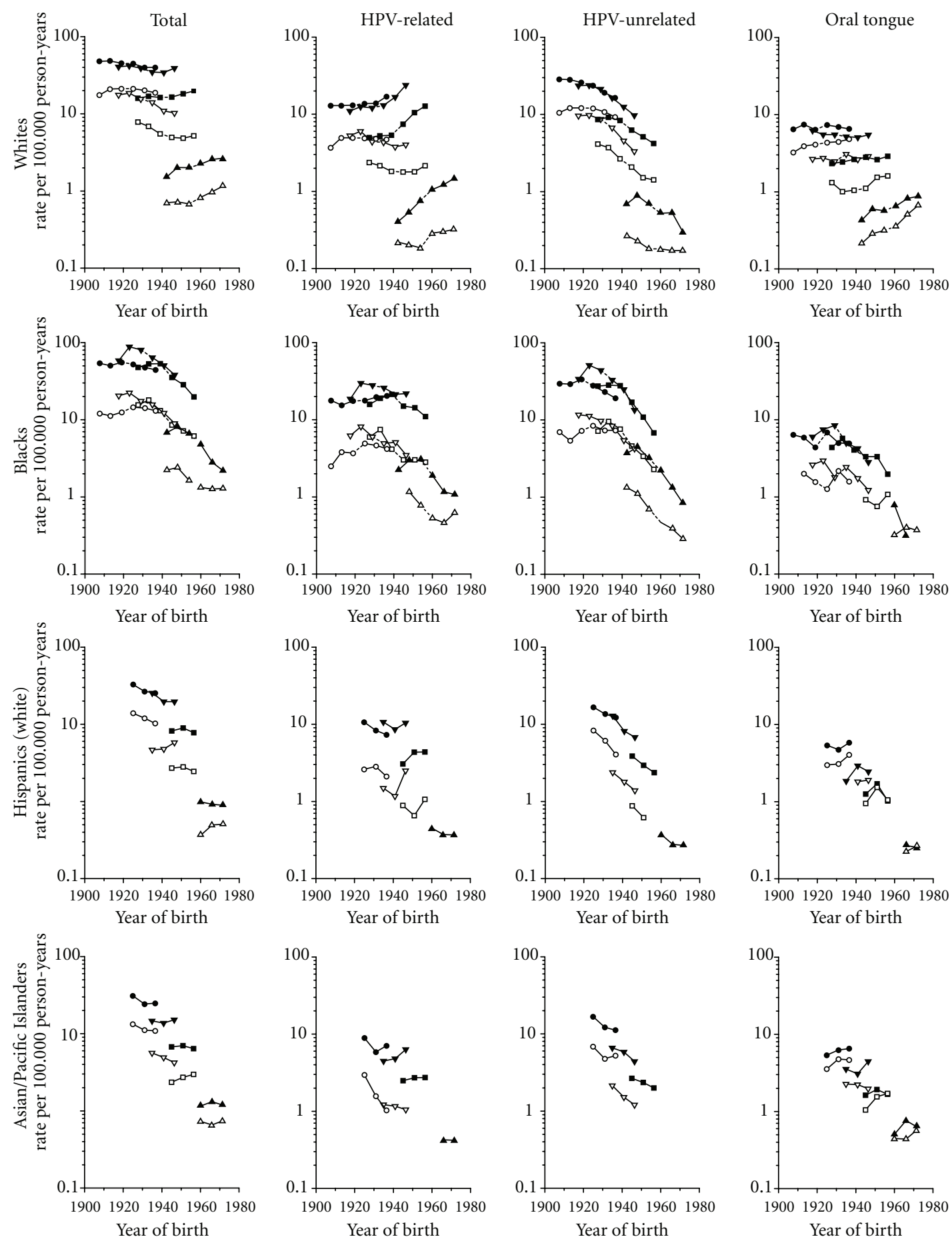

$$
\begin{aligned}
& \text { Males Females }
\end{aligned}
$$

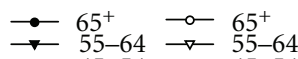

$$
\begin{aligned}
& \because \quad 45-54 \because 45-54
\end{aligned}
$$

FIGURE 2: Trends in age-specific squamous cell carcinoma of the oral cavity and pharynx incidence rates by race/ethnicity, subsite, and gender for whites and blacks SEER 9 1975-1979 to 1986-1991 and for whites (non-Hispanic), blacks, Hispanics (white), and Asian/PIs SEER 13 1992-2008 according to year of birth (excludes lip, salivary glands, and nasopharynx) (rates age-adjusted within age group). 

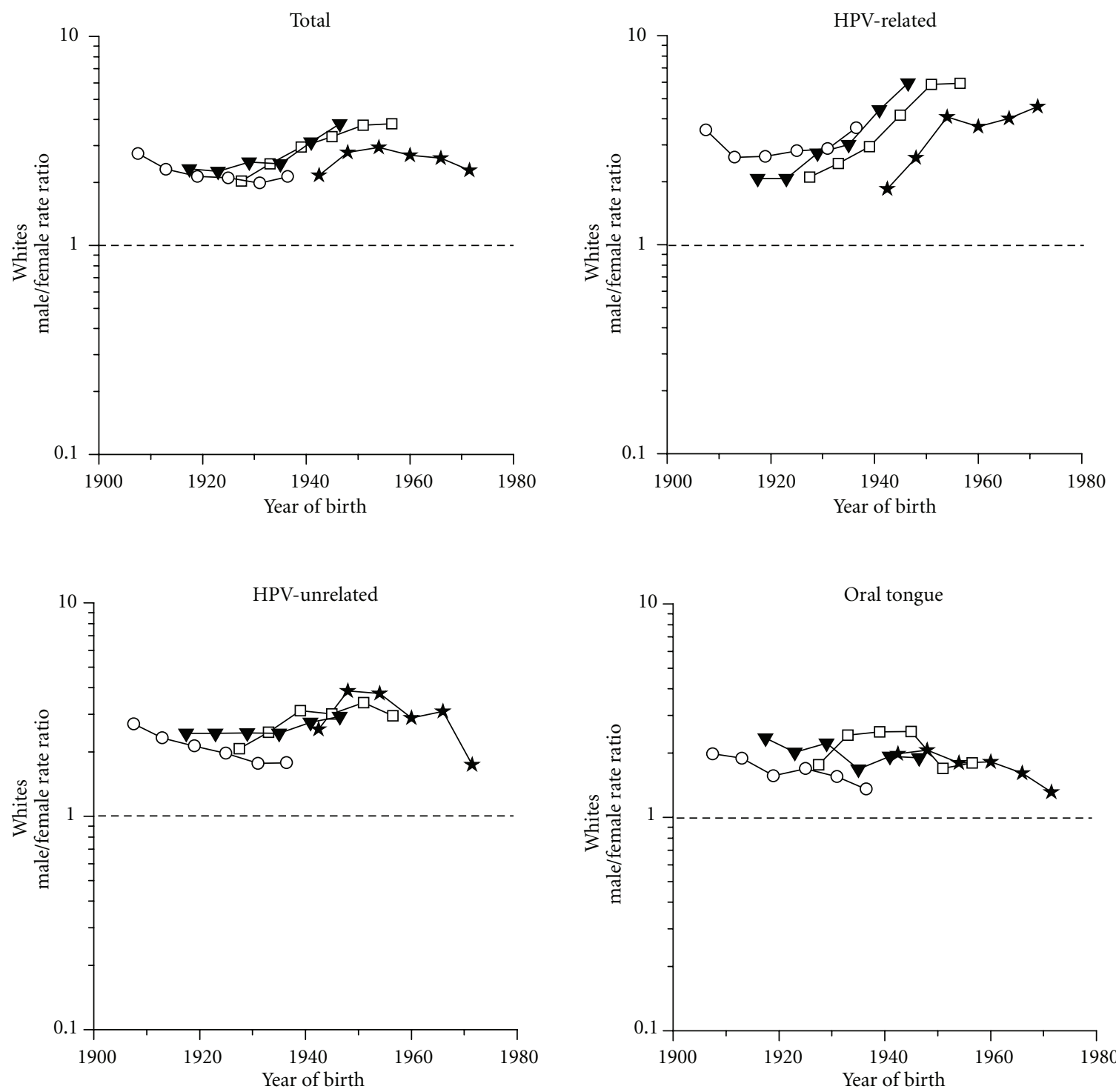

$\begin{array}{llll}-0 & 65^{+} & -\square & 45-54 \\ \neg & 55-64 & \star & 25-44\end{array}$

FIGURE 3: Trends in age-specific squamous cell carcinoma of the oral cavity and pharynx male/female incidence rate ratios among whites by subsite SEER 9 1975-1979 to 1986-1991 and whites (non-Hispanic) SEER 13 1992-2008 according to year of birth (excludes lip, salivary glands, and nasopharynx).

ranging between 2 and 3 among those born prior to the mid1940s, rising to between 3 and 4 among those born during the 1950s and falling back to 3 or lower subsequently. Oral tongue cancer IRRs were all $<3$; among both the oldest and the youngest age groups, the IRRs decreased from about 2 to 1.3-1.4 over the study period.

\section{Discussion}

In this study, we analyzed temporal trends of total OCP, presumed HPV-related, presumed HPV-unrelated, and oral tongue cancer among US men and women of white, black, Hispanic, Asian, and AI/AN ethnicity. Our study, which highlights changing patterns by gender, is based on the same categorization of anatomic subsites as presumed HPVrelated or not used in two recent publications [11, 12], and we extend these findings by classifying cancer of the oral tongue separately from other HPV-unrelated sites, incorporating cases diagnosed through 2008, increasing the study population by including data from SEER 13 , classifying whites as Hispanic or non-Hispanic, and presenting MF IRRs overall and by year of birth.

Similar to other studies, we found overall OCPC incidence rates among men to be $2-4$ times those among women of all race/ethnicities, and in recent decades, they varied from 4 or greater for HPV-related sites to 2-3 for HPVunrelated sites, and $1-3$ for oral tongue cancer. The MF IRR for HPV-related sites doubled among whites from 2.4 to 4.8 over the study period, due especially to rapidly rising rates 
of HPV-related OCPC among white men, particularly those born since 1940. Consistent decreases in the MF IRRs for HPV-unrelated sites were observed for whites (from 2.4 to 2.1) and blacks (from 4.0 to 2.8 ), due to rapidly declining rates especially among black men born since 1940. The IRRs for oral tongue cancer generally declined among all ethnic groups due especially to rising rates among younger white, Hispanic, and Asian women. Total OCPC trends varied markedly over the period 1985-2008. The age-adjusted rates for HPV-related cancers rose 3\% per year among white males, for HPV-unrelated cancers declined 3\% per year among whites of both genders and black females and 5\% per year among black males, and for oral tongue cancer rose $1 \%$ per year among white females.

The declines seen in HPV-unrelated cancer for all race/ ethnic-sex groups are consistent with a pathway mainly driven by alcohol and tobacco (potentially related to p53 or other molecular alterations [23]) that parallel the reductions in US cigarette smoking prevalence [24] and may also reflect decreases in national per capita alcohol consumption, especially the use of hard liquor [25]. The steep declines in SCC of the OCP and racial/gender patterns seen here resemble the patterns for SCC of the esophagus, which also reflect the trends in cigarette smoking prevalence and alcohol consumption [26].

Among white males, the much smaller declines in the incidence of total OCPC appear to be driven by the rising incidence of HPV-related cancers, in contrast to the declines in HPV-unrelated cancers [27-29]. A number of studies have documented the increasing prevalence of HPV in oropharyngeal cancers during the past 30 years. In the US, the prevalence of HPV in oropharyngeal cancers rose from $33 \%$ during the 1980 s to $70 \%$ in the 1990 s and $82 \%$ during 2000-2004, based on 72 cases assayed in Colorado [10] and from $16 \%$ during the 1980 s to $73 \%$ during the 2000s based on 271 cases from SEER tissue repositories in Hawaii, Iowa, and Los Angeles [13]. During the 2000s, several groups have reported HPV prevalence rates among oropharynx, tongue, and tonsil cases ranging from $63 \%$ to $92 \%$, whereas the prevalence among other oral cancers has been $20 \%$ or less [30-32]. Thus, our assignment of presumed HPV status based on anatomic subsite certainly included non-HPV-related cases, more so during the earlier years compared to more recently. The incidence data suggest that the proportions also varied by gender, racial/ethnic group, and age.

HPV is a very common sexually transmitted infection in the United States [33]. Data from the 2003-2004 National Health and Nutrition Examination Survey (NHANES) relinebreak vealed that the seroprevalence of any HPV types $6,11,16$, or 18 (types in the quadrivalent vaccine) among persons aged $14-59$ years was $22.4 \%$ overall, higher among females $(32.5 \%)$ than males $(12.1 \%)$, peaked at $42.0 \%$ among females aged 30-39 years and $18.0 \%$ among males aged 50-59 years, and was associated with lifetime number of sexual partners, especially among females [34]. About $90 \%$ of both females and males aged 25-44 ever had oral sex with an opposite-sex partner, based on data from the 2006-2008 National Survey of Family Growth (NSFG) [35].
The prevalence of oral infection with any of 37 HPV DNA types during 2009-2010 was 6.9\% among those aged 1469 , significantly higher among men $(10.1 \%)$ than women $(3.6 \%)$, and increased with the number of sexual partners and cigarettes smoked per day, based on NHANES data [36].

Similar to other recent studies of oral tongue cancer $[16,37]$, we found increasing rates among young white adults aged 25-44 that were more rapid for women than for men, and some suggestion of similar patterns among blacks, Hispanics, and Asians. The causal pathway for oral tongue cancer in these patients is not known, but it does not appear to be related to use of alcohol or tobacco [16] or to the presence of HPV $[16,37]$. Its unique age- and sexspecific incidence patterns suggest that it may have a different etiology from other OCPCs [37] including other oncogenic viruses, bacteria, and lifestyle and environmental factors.

A limitation of our descriptive study is that HPV status is based on a categorization by anatomic subsite and thus likely resulted in some misclassification of actual HPV status. It has been suggested that HPV-related base of the tongue cancer may have been misclassified as oral tongue $[11,16]$; however, the MF IRRs appear to be very different [37]. Cases without a particular site of the tongue specified (coded C02.9= tongue, not otherwise specified) accounted for about $15 \%$ of oral tongue cases among whites during 1975-2008, and the proportion rose from $13 \%$ during $1975-1979$ to $17 \%$ during 2004-2008. To the extent that some of these may have been HPV-related, then the observed increases in HPV-related cancers among white males have been underestimated. The observed increases in HPV-unrelated oral tongue cancer among white females may have been overstated; however, rates for each specific tongue subsite as well as tongue, NOS, all rose.

In summary, our large numbers based on SEER 13 allowed us to plot race-, sex-, and age-specific incidence rates of OCPC by HPV category and year of birth and to carry out a detailed analysis of oral tongue cancers. The significant decreases in total OCPC incidence seen for most race/sex groups reflect reductions in smoking prevalence due to public health efforts aimed at cessation and lowering initiation rates as well as decreases in alcohol consumption. The increasing rates of cancer of the oral tongue especially among young men and women is of growing concern and suggest the need for dedicated research into the epidemiology of these understudied tumors; the narrowing of the gender differences apparent across all the racial/ethnic groups provides a clue worthy of pursuit. The much smaller decline in total OCPC incidence and recent increases among white men born since the mid-1940s appear related to the rapidly rising rates of $\mathrm{HPV}$-related cancers and may reflect changes in sexual practices since the mid-1960s. The potential impact of HPV vaccination recently approved for both girls and boys on these trends is unknown, but changes are likely many years in the future. Dentists and physicians need to be aware of the notable and significant rise in rates of cancers of the oral tongue and HPV-related cancers of the oropharynx, especially among young men and women, 
and ensure that these new at-risk groups receive routine oral cancer screening.

\section{Financial Support}

This study was supported by the Intramural Program of the Division of Cancer Epidemiology and Genetics, National Cancer Institute (NCI), National Institutes of Health.

\section{References}

[1] N. Howlader, A. M. Noone, M. Krapcho et al., "SEER Cancer Statistics Review, 1975-2008," National Cancer Institute, Bethedsa, Md, USA, 2011, http://www.seer.cancer.gov/csr/ 1975_2008/.

[2] B. A. Kohler, E. Ward, B. J. McCarthy et al., "Annual report to the nation on the status of cancer, 1975-2007, featuring tumors of the brain and other nervous system," Journal of the National Cancer Institute, vol. 103, no. 9, pp. 714-736, 2011.

[3] E. M. Sturgis, Q. Wei, and M. R. Spitz, "Descriptive epidemiology and risk factors for head and neck cancer," Seminars in Oncology, vol. 31, no. 6, pp. 726-733, 2004.

[4] S. T. Mayne, D. E. Morse, and D. M. Winn, "Cancers of the oral cavity and pharynx," in Cancer Epidemiology and Prevention, D. Schottenfeld and J. F. Fraumeni Jr., Eds., pp. 674-696, Oxford University Press, New York, NY, USA, 3rd edition, 2006.

[5] M. L. Gillison, "Human papillomavirus-associated head and neck cancer is a distinct epidemiologic, clinical, and molecular entity," Seminars in Oncology, vol. 31, no. 6, pp. 744-754, 2004.

[6] IARC, Monographs on the Evaluation of Carcinogenic Risks to Humans, vol. 90 of Human Papillomaviruses, World Health Organization, International Agency for Research on Cancer, Lyon, France, 2007.

[7] S. Marur, G. D'Souza, W. H. Westra, and A. A. Forastiere, "HPV-associated head and neck cancer: a virus-related cancer epidemic," The Lancet Oncology, vol. 11, no. 8, pp. 781-789, 2010.

[8] M. Frisch, H. Hjalgrim, A. B. Jaeger, and R. J. Biggar, "Changing patterns of tonsillar squamous cell carcinoma in the United States," Cancer Causes and Control, vol. 11, no. 6, pp. 489-495, 2000.

[9] C. H. Shiboski, B. L. Schmidt, and R. C. Jordan, "Tongue and tonsil carcinoma: increasing trends in the U.S. population ages 20-44 years," Cancer, vol. 103, no. 9, pp. 1843-1849, 2005.

[10] J. A. Ernster, C. G. Sciotto, M. M. O’Brien et al., "Rising incidence of oropharyngeal cancer and the role of oncogenic human papilloma virus," Laryngoscope, vol. 117, no. 12, pp. 2115-2128, 2007.

[11] A. K. Chaturvedi, E. A. Engels, W. F. Anderson, and M. L. Gillison, "Incidence trends for human papillomavirus-related and -unrelated oral squamous cell carcinomas in the United States," Journal of Clinical Oncology, vol. 26, no. 4, pp. 612619, 2008.

[12] L. M. Brown, D. P. Check, and S. S. Devesa, "Oropharyngeal cancer incidence trends: diminishing racial disparities," Cancer Causes and Control, vol. 22, no. 5, pp. 753-763, 2011.

[13] A. K. Chaturvedi, E. A. Engels, R. M. Pfeiffer et al., "Human papillomavirus and rising oropharyngeal cancer incidence in the United States," Journal of Clinical Oncology, vol. 29, no. 32, pp. 4294-4301, 2011.
[14] V. Mehta, G. P. Yu, and S. P. Schantz, "Population-based analysis of oral and oropharyngeal carcinoma: changing trends of histopathologic differentiation, survival and patient demographics," Laryngoscope, vol. 120, no. 11, pp. 2203-2212, 2010.

[15] A. B. Ryerson, E. S. Peters, S. S. Coughlin et al., "Burden of potentially human papillomavirus-associated cancers of the oropharynx and oral cavity in the US, 1998-2003," Cancer, vol. 113, no. 10, pp. 2901-2909, 2008.

[16] S. C. Patel, W. R. Carpenter, S. Tyree et al., "Increasing incidence of oral tongue squamous cell carcinoma in young white women, age 18 to 44 years," Journal of Clinical Oncology, vol. 29, no. 11, pp. 1488-1494, 2011.

[17] Surveillance, Epidemiology, and End Results (SEER) Program, "SEER* Stat Database: Incidence-SEER 13 Regs LimitedUse, Nov 2010 Sub (1992-2008) (Katrina/Rita Population Adjustment) - Linked to County Attributes-Total U.S., 1969-2009 Counties," National Cancer Institute, DCCPS, Surveillance Research Program, Cancer Statistics Branch, April 2011.

[18] Surveillance, Epidemiology, and End Results (SEER) Program, "SEER*Stat Database: Incidence-SEER 9 Regs LimitedUse, Nov 2010 Sub (1973-2008) (Katrina/Rita Population Adjustment)—Linked to County Attributes_-Total U.S., 1969-2009 Counties," National Cancer Institute, DCCPS, Surveillance Research Program, Cancer Statistics Branch, April 2011.

[19] A. Fritz, C. Percy, K. Jack et al., International Classification of Diseases for Oncology, World Health Organization, Geneva, Switzerland, 3rd edition, 2000.

[20] Surveillance Research Program, National Cancer Institute, SEER* Stat software, http://www.seer.cancer.gov/seerstat/.

[21] R. Tiwari, L. Clegg, and Z. Zou, "Efficient interval estimation for age-adjusted cancer rates," Statistical Methods in Medical Research, vol. 15, no. 6, pp. 547-569, 2006.

[22] S. S. Devesa, J. Donaldson, and T. Fears, "Graphical presentation of trends in rates," American Journal of Epidemiology, vol. 141, no. 4, pp. 300-304, 1995.

[23] G. D'Souza, A. R. Kreimer, R. Viscidi et al., "Case-control study of human papillomavirus and oropharyngeal cancer," The New England Journal of Medicine, vol. 356, no. 19, pp. 1944-1956, 2007.

[24] E. M. Sturgis and P. M. Cinciripini, "Trends in head and neck cancer incidence in relation to smoking prevalence: an emerging epidemic of human papillomavirus-associated cancers?" Cancer, vol. 110, no. 7, pp. 1429-1435, 2007.

[25] L. M. Brown, "Epidemiology of alcohol-associated cancers," Alcohol, vol. 35, no. 3, pp. 161-168, 2005.

[26] L. M. Brown and S. S. Devesa, "Epidemiologic trends in esophageal and gastric cancer in the United States," Surgical Oncology Clinics of North America, vol. 11, no. 2, pp. 235-256, 2002.

[27] M. L. Gillison, W. M. Koch, R. B. Capone et al., "Evidence for a causal association between human papillomavirus and a subset of head and neck cancers," Journal of the National Cancer Institute, vol. 92, no. 9, pp. 709-720, 2000.

[28] R. I. Haddad and D. M. Shin, "Recent advances in head and neck cancer," The New England Journal of Medicine, vol. 359, no. 11, pp. 1143-1096, 2008.

[29] M. L. Gillison, "Current topics in the epidemiology of oral cavity and oropharyngeal cancers," Head and Neck, vol. 29, no. 8, pp. 779-792, 2007.

[30] C. Fakhry, W. H. Westra, S. Li et al., "Improved survival of patients with human papillomavirus-positive head and 
neck squamous cell carcinoma in a prospective clinical trial," Journal of the National Cancer Institute, vol. 100, no. 4, pp. 261-269, 2008.

[31] G. D’Souza, H. H. Zhang, W. D. D’Souza, R. R. Meyer, and M. L. Gillison, "Moderate predictive value of demographic and behavioral characteristics for a diagnosis of HPV16-positive and HPV16-negative head and neck cancer," Oral Oncology, vol. 46, no. 2, pp. 100-104, 2010.

[32] D. W. Kingma, R. A. Allen, W. Moore et al., "HPV genotype distribution in oral and oropharyngeal squamous cell carcinoma using seven in vitro amplification assays," Anticancer Research, vol. 30, no. 12, pp. 5099-5104, 2010.

[33] S. Hariri, E. R. Unger, M. Stemberg et al., "Prevalence of genital human papillomavirus among females in the United States, the National Health and Nutrition Examination Survey, 2003-2006," Journal of Infectious Diseases, vol. 204, no. 4, pp. 566-573, 2011.

[34] L. E. Markowitz, M. Sternberg, E. F. Dunne, G. McQuillan, and E. R. Unger, "Seroprevalence of human papillomavirus types 6, 11, 16, and 18 in the United States: National Health and Nutrition Examination Survey 2003-2004," Journal of Infectious Diseases, vol. 200, no. 7, pp. 1059-1067, 2009.

[35] A. Chandra, W. D. Mosher, C. Copen, and C. Sionean, "Sexual behavior, sexual attraction, and sexual identity in the United States: data from the 2006-2008 National Survey of Family Growth," National Health Statistics Reports, vol. 3, no. 36, pp. $1-36,2011$.

[36] M. L. Gillison, T. Broutian, R. K. L. Pickard et al., "Prevalence of oral HPV infection in the United States 2009-2010," Journal of the American Medical Association, vol. 307, no. 7, pp. 693$703,2012$.

[37] N. F. Saba, M. Goodman, K. Ward et al., "Gender and ethnic disparities in incidence and survival of squamous cell carcinoma of the oral tongue, base of tongue, and tonsils: a Surveillance, Epidemiology and End Results program-based analysis," Oncology, vol. 81, no. 1, pp. 12-20, 2011. 


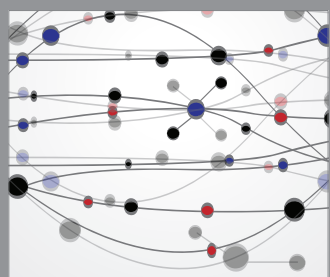

The Scientific World Journal
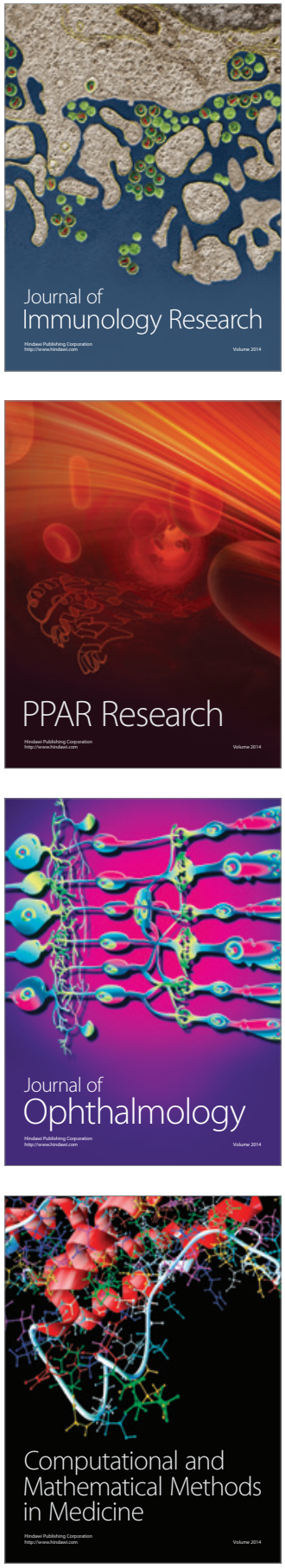

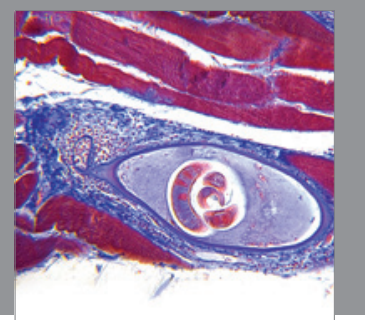

Gastroenterology

Research and Practice
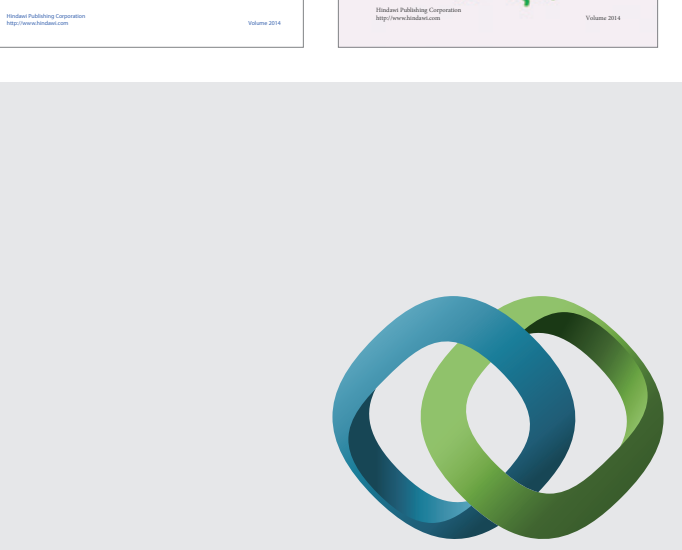

\section{Hindawi}

Submit your manuscripts at

http://www.hindawi.com
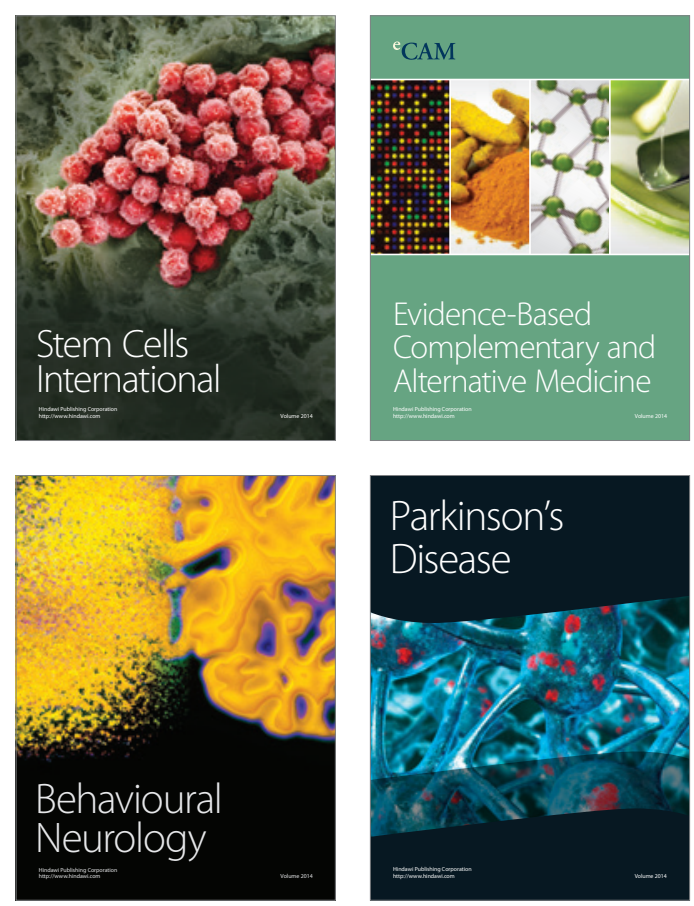

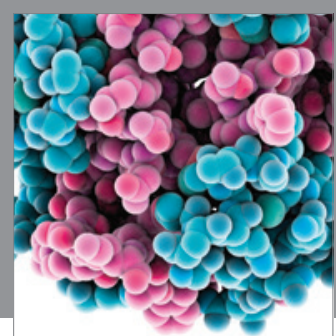

Journal of
Diabetes Research

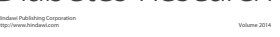

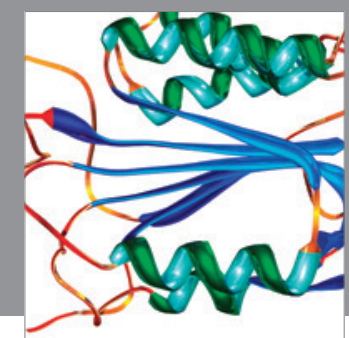

Disease Markers
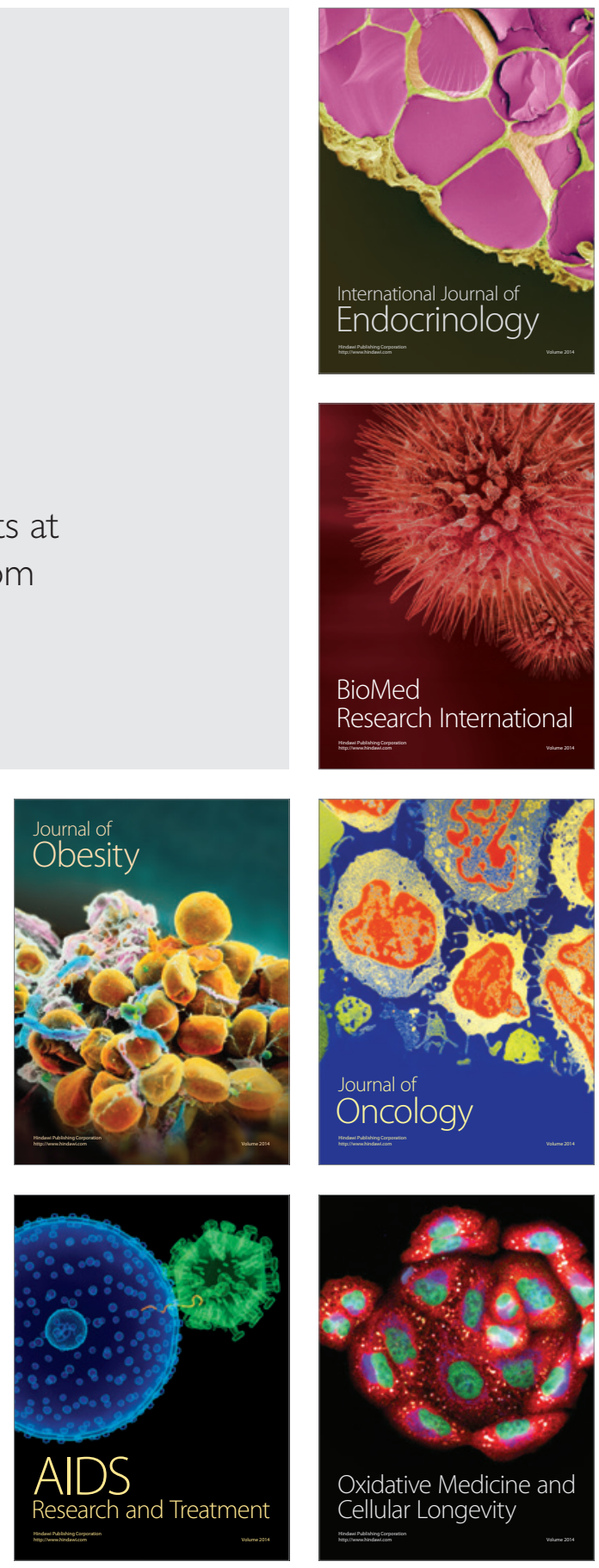